\title{
Constraining relativistic protons and magnetic fields in galaxy clusters through radio and $\gamma$-ray observations: the case of A2256 (Research Note)
}

\author{
G. Brunetti
}

\author{
INAF - Istituto di Radioastronomia, via P. Gobetti 101, 40129 Bologna, Italy \\ e-mail: brunetti@ira.inaf.it
}

Received 25 August 2009 / Accepted 18 September 2009

\begin{abstract}
Giant radio halos are the most relevant examples of diffuse synchrotron emission from galaxy clusters. A number of these sources have very steep spectra, of spectral index $\alpha \geq 1.5-1.6\left(F(v) \propto v^{-\alpha}\right)$, and are ideal targets for testing current models of the origin of the relativistic particles. A2256 hosts the nearest radio halo with a very steep spectrum, of $\alpha=1.61$, and a very large population of relativistic protons in the cluster would be necessary if the halo were produced by synchrotron emission from secondary particles. In this case, the $0.1-1 \mathrm{GeV} \gamma$-ray luminosity is expected to be 10-20 times higher than that of clusters hosting radio halos of similar radio power at $\mathrm{GHz}$ frequencies but with spectra more typical of the presently observed halo population, $\alpha \sim 1.2$. Based on these assumptions, future FERMI/GLAST observations are expected to detect A2256, provided that the magnetic field in the central cluster region is $\leq 10-15 \mu \mathrm{G}$. We show that this will provide a prompt test of hadronic models for the origin of radio halos, and complementary constraints on both the cluster magnetic field and the physics of particle acceleration mechanisms.
\end{abstract}

Key words. radiation mechanisms: non-thermal - galaxies: clusters: general - radio continuum: general - gamma rays: theory

\section{Introduction}

Galaxy clusters are the largest gravitationally bound objects in the Universe. During cluster mergers, energy may be channelled into both the amplification of the magnetic fields (Dolag et al. 2005; Ryu et al. 2008) and the acceleration of relativistic primary electrons (CRe) and protons (CRp) via shocks and turbulence (e.g., Ensslin et al. 1998; Sarazin 1999; Blasi 2001; Ryu et al. 2003; Gabici \& Blasi 2003; Pfrommer et al. 2006; Brunetti \& Lazarian 2007). CRp have long lifetimes and remain confined within clusters for a Hubble time (Völk et al. 1996; Berezinsky et al. 1997; Ensslin et al. 1997). They are expected to be the dominant non-thermal particle component in the ICM and should produce secondary particles due to collisions with thermal protons (e.g., Blasi et al. 2007, for a review).

Direct evidence of magnetic fields and relativistic particles, mixed with the thermal intracluster medium (ICM) comes from radio observations that detect $\mathrm{Mpc}$-sized diffuse radio sources, radio halos and relics, in a fraction of X-ray luminous galaxy clusters in merging phase (e.g., Ferrari et al. 2008, for a review). Extended and fairly regular diffuse synchrotron emission, in the form of giant radio halos, may be produced by secondary electrons injected during proton-proton collisions (hadronic models, e.g., Dennison 1980; Blasi \& Colafrancesco 1999; Pfrommer \& Ensslin 2004), or by assuming that relativistic electrons are re-accelerated in-situ by MHD turbulence generated in the ICM during cluster-cluster mergers (re-acceleration models, e.g., Brunetti et al. 2001, 2004; Petrosian 2001; Fujita et al. 2003; Cassano \& Brunetti 2005). Unavoidable $\gamma$-ray emission, due to the decay of the neutral pions generated by proton-proton collisions, is expected in the context of hadronic models (e.g., Blasi \& Colafrancesco 1999; Miniati 2003; Pfrommer \& Ensslin 2004). Some $\gamma$-ray emission is also expected by re-acceleration models that account for the general situation where relativistic protons and electrons (including secondaries) interact with MHD turbulence (Brunetti \& Blasi 2005; Brunetti 2009). Those halos with very steep spectrum are suitable targets for constraining models and their properties are most consistent with a turbulent re-acceleration scenario (e.g., Brunetti et al. 2008). Indeed clusters hosting radio halos with very steep spectrum should contain a very large population of CRp according to the hadronic scenario; this also implies an unavoidably large amount of $\gamma$-ray emission from these clusters.

Only upper limits to the $\gamma$-ray emission from clusters have been so far obtained (Reimer et al. 2003; Aharonian et al. 2009 b), providing in some cases a fairly stringent constraint on the energy density of CRp, $<10 \%$ of that of the thermal ICM (Aharonian et al. 2009a). The FERMI/GLAST telescope will soon provide tighter constraints on both the $\gamma$-ray properties of clusters and the energy density of CRp.

The radio halo in A2256 is our most nearby steep-spectrum halo, and we show that the incoming FERMI/GLAST data will provide a prompt test of the hadronic scenario and allow us to constrain the cluster-magnetic field. We assume a $\Lambda \mathrm{CDM}$ cosmology $\left(H_{0}=70 \mathrm{~km} \mathrm{~s}^{-1} \mathrm{Mpc}^{-1}, \Omega_{\mathrm{m}}=0.3, \Omega_{\Lambda}=0.7\right)$ throughout the paper.

\section{The cluster Abell 2256}

Abell 2256 is a massive galaxy cluster at $z=0.058$, with a $0.1-2.4 \mathrm{keV} X$-ray luminosity $L_{X} \simeq 3.8 \times 10^{44} \mathrm{erg} / \mathrm{s}$ (e.g., Ebeling et al. 1996). The dynamical state of A2256 is complex and is thought to consist of at least three merging systems based on optical velocity dispersion (Berrington et al. 2002; Miller et al. 2003). A complex dynamical status of A2256 is also 
suggested by X-ray observations that detect two separate peaks in the X-ray surface brightness distribution corresponding to the primary cluster and the secondary subcluster, which is infalling onto the primary from the northeast (Briel et al. 1991; Sun et al. 2002).

Radio observations have detected complex diffuse emission on large scales (Bridle et al. 1979; Rottgering et al. 1994; Clarke \& Ensslin 2006; Brentjens 2008) consisting of a bright relic, northwest of the cluster center and a fainter steep-spectrum Mpcscale radio halo in the cluster central region. Deep observations at 1400 and $300 \mathrm{MHz}$ detect diffuse radio-halo emission out to a distance from the cluster center $\sim 1.5 r_{\mathrm{c}} \approx 520 \mathrm{kpc}$ (Clarke \& Ensslin 2006; Brentjens 2008). A detailed spectral analysis of the halo emission derived an integrated spectral index between $0.3-1.4 \mathrm{GHz}$ of $\alpha=1.61\left(F(v) \propto v^{-\alpha}\right)$, once the contribution from embedded discrete radio sources had been subtracted (Brentjens 2008).

\section{Hadronic models: formalism}

The decay chain that we consider for the injection of secondary particles in the ICM due to $p-p$ collisions is (Blasi \& Colafrancesco 1999)

$p+p \rightarrow \pi^{0}+\pi^{+}+\pi^{-}+$anything

$\pi^{0} \rightarrow \gamma \gamma$

$\pi^{ \pm} \rightarrow \mu+v_{\mu} \quad \mu^{ \pm} \rightarrow \mathrm{e}^{ \pm} v_{\mu} v_{\mathrm{e}}$

which is a threshold reaction that requires protons with kinetic energies higher than $T_{\mathrm{p}} \approx 300 \mathrm{MeV}$.

The injection rate of pions is

$Q_{\pi}^{ \pm, \mathrm{o}}\left(E_{\pi^{ \pm, \mathrm{o}}}, t\right)=n_{\mathrm{th}} c \int_{p_{*}} \mathrm{~d} p N_{\mathrm{p}}(p, t) \beta_{\mathrm{p}} \frac{F_{\pi}\left(E_{\pi}, E_{\mathrm{p}}\right) \sigma^{ \pm, \mathrm{o}}(p)}{\sqrt{1+\left(m_{\mathrm{p}} c / p\right)^{2}}}$,

where $n_{\mathrm{th}}$ is the number density of thermal protons, and $F_{\pi}$ is the spectrum of pions from the collision between a CRp of energy $E_{\mathrm{p}}$ and thermal protons (taken from Brunetti \& Blasi 2005). The inclusive cross-section, $\sigma(p)$, is taken from the fitting formulae of Dermer (1986b), which allow us to describe separately the rates of generation of $\pi^{-}, \pi^{+}$, and $\pi^{o}$, and $p_{*}=\max \left\{p_{\mathrm{tr}}, p_{\pi}\right\}$, where $p_{\text {tr }}$ is the threshold momentum of protons.

The spectrum of $\gamma$-rays produced by the decay of the secondary $\pi^{o}$ is (e.g., Dermer 1986a,b; Blasi \& Colafrancesco 1999)

$Q_{\gamma}\left(E_{\gamma}\right)=2 \int_{E_{\min }}^{E_{\mathrm{p}}^{\max }} \frac{Q_{\pi^{o}}\left(E_{\pi^{o}}\right)}{\sqrt{E_{\pi}^{2}-m_{\pi}^{2} c^{4}}} \mathrm{~d} E_{\pi}$,

where $E_{\min }=E_{\gamma}+1 / 4 m_{\pi}^{2} c^{4} / E_{\gamma}$.

Charged pions decay into muons and secondary pairs (electrons and positrons). Based on the assumption that secondaries are not accelerated by other mechanisms, their spectrum approaches a stationary distribution because of the competition between injection and energy losses (e.g., Dolag \& Ensslin 2000)

$N_{\mathrm{e}}^{ \pm}(p)=\frac{1}{\left|\left(\frac{\mathrm{d} p}{\mathrm{~d} t}\right)_{\text {loss }}\right|} \int_{\mathrm{p}}^{p_{\max }} Q_{\mathrm{e}}^{ \pm}(p) \mathrm{d} p$,

where $Q_{\mathrm{e}}^{ \pm}$is the injection rate of secondaries (e.g., Blasi \& Colafrancesco 1999; Moskalenko \& Strong 1998), and radiative

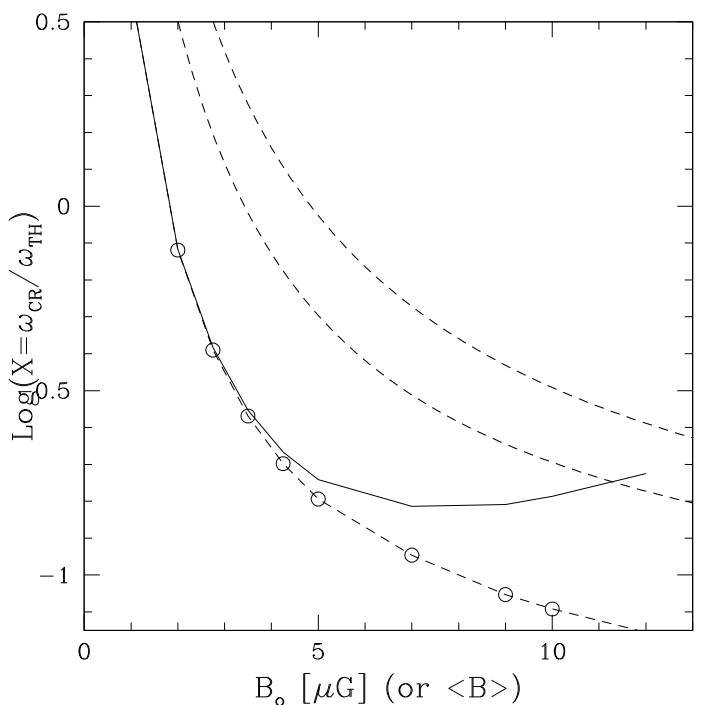

Fig. 1. Ratio of relativistic CRp to thermal energy densities (for $r \leq$ $1.5 r_{\mathrm{c}}$ ) for the steep (dashed line, open circles) and flat models (red dashed lines) as a function of $\langle B\rangle$ (steep model) and $B_{\mathrm{o}}$ (flat model; $b=0.5,1$ from bottom to top). The ratio of $\mathrm{CRp}+B$ to thermal energy densities is shown for the steep model (solid line).

losses, which are dominant for $\gamma>10^{3}$ electrons in the ICM, are given by (e.g., Sarazin 1999)

$\left|\left(\frac{\mathrm{d} p}{\mathrm{~d} t}\right)_{\mathrm{loss}}\right| \simeq 3.3 \times 10^{-32}\left(\frac{p / m_{\mathrm{e}} c}{300}\right)^{2}\left[\left(\frac{B_{\mu \mathrm{G}}}{3.2}\right)^{2}+(1+z)^{4}\right]$.

Assuming a power law distribution of CRp, $N_{\mathrm{p}}(p)=K_{\mathrm{p}} p^{-s}$, the spectrum of secondaries at high energies, $\gamma>10^{3}$, is $N_{\mathrm{e}}(p) \propto p^{-(s+1)} \mathcal{F}(p)$, where $\mathcal{F}$ accounts for the log-scaling of the $p-p$ cross-section at high energies and causes the spectral shape to be slightly flatter than $p^{-(s+1)}$ (e.g., Brunetti \& Blasi 2005). The synchrotron spectrum from secondary $\mathrm{e}^{ \pm}$is (e.g., Rybicky \& Lightman 1979)

$$
\begin{aligned}
J_{\mathrm{syn}}(v) & =\sqrt{3} \frac{\mathrm{e}^{3}}{m_{\mathrm{e}} c^{2}} B \int_{0}^{\pi / 2} \mathrm{~d} \theta \sin ^{2} \theta \int \mathrm{d} p N_{\mathrm{e}}(p) F\left(\frac{v}{v_{\mathrm{c}}}\right) \\
& \simeq C(\alpha, T) X n_{\mathrm{th}}^{2} \frac{B^{1+\alpha}}{B^{2}+B_{\mathrm{cmb}}^{2}} v^{-\alpha},
\end{aligned}
$$

where $C$ is a constant, $X=\omega_{\mathrm{CR}} / \omega_{\mathrm{TH}}$ is the ratio of the energy densities of CRp to thermal protons, $F$ is the synchrotron kernel, $v_{\mathrm{c}}$ is the critical frequency, and $\alpha \simeq s / 2-\Delta$, where $\Delta \sim 0.1-0.15$ is due to the log-scaling of the cross-section.

\section{Results}

We show that the steep spectrum of the halo in A2256 (Sect. 2) allows a prompt test of hadronic models and a constraint of the magnetic field in the ICM.

We assume that the radio halo is produced by synchrotron emission from secondary electrons, where the observed synchrotron spectral index, $\alpha=1.61$, implies that $s=3.4-3.5$. The parameters for the thermal ICM distribution in A2256, $n_{\mathrm{o}}$, $T, r_{\mathrm{c}}$, and $\beta$, were taken from Henry et al. (1993) and Myers et al. (1997).

We first adopt a steep model that assumes a constant ratio of the energy density of CRp to thermal protons, $\omega_{\mathrm{CR}} / \omega_{\mathrm{TH}}=X$, 

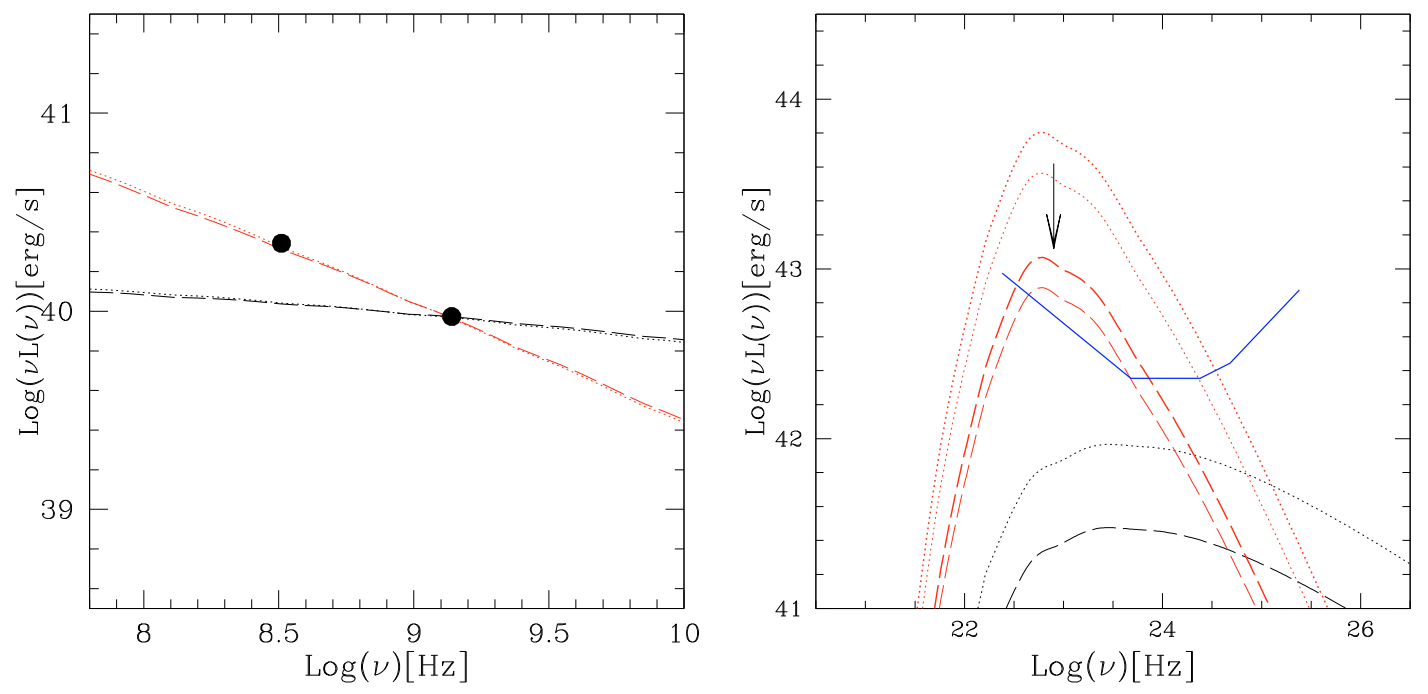

Fig. 2. Radio (left) and ( $\pi^{o}$-decay) $\gamma$-rays from $r \leq 3 r_{\mathrm{c}}$ (right) from A2256 assuming $b=0.5$ and $B_{0}=2.65$ (dotted lines) and $10 \mu \mathrm{G}$ (dashed lines). Calculations are shown for $s=3.5$ (red lines) in the case of the flat (thick lines) and steep model (thin lines). Results for a steep model with $s=2.4$ are also shown for comparison. Monochromatic radio luminosities at 330 and $1400 \mathrm{MHz}$ (filled points), the EGRET upper limit (arrow), and the FERMI/GLAST reference sensitivity (solid-blue line) are also shown.

and model the halo region with a homogeneus sphere of radius $R_{\mathrm{H}} \sim 1.5 r_{\mathrm{c}}$ and a volume-averaged field (weighted for synchrotron emissivity) $\langle B\rangle$. For relativistic CRp only, the value of $X$ required to reproduce the observed synchrotron spectrum is shown in Fig. 1 as a function of $\langle B\rangle$. We find that $\langle B\rangle \leq 2 \mu \mathrm{G}$ can be excluded since the CRp energy density becomes higher than the cluster thermal budget. For stronger magnetic fields, $\langle B\rangle \geq 4-5 \mu \mathrm{G}, \omega_{\mathrm{CR}} \leq 0.2 \omega_{\mathrm{TH}}$ and the non-thermal component is dominated by the magnetic field. The non-thermal energy content reaches a minimum, $\sim 0.16 \omega_{\mathrm{TH}}$, for $\langle B\rangle \approx 7-9 \mu \mathrm{G}$, which represents the minimum energy condition for hadronic models (Pfrommer \& Ensslin 2004). If we do not restrict ourselves to relativistic CRp and also include subrelativistic CRp, because of the very steep spectrum, the required energy budget of CRp is much greater than that in Fig. $1, \omega_{\mathrm{CR}} \propto p_{\min }^{-s+3}$.

We assume a spatial profile of the magnetic field $B=$ $B_{\mathrm{o}}\left(\frac{n_{\mathrm{th}}}{n_{\mathrm{o}}}\right)^{b}$ (e.g., Govoni \& Feretti 2004) and find that the steep model produces a radio-halo brightness profile that decreases by a factor $25-40$ at $r \sim 1.5 r_{\mathrm{c}}$, by adopting $b=0.5-1$ and $B_{\mathrm{o}} \geq 5 \mu \mathrm{G}$. This is inconsistent with the observed profile that drops, at the same distance, by only a factor 5-8 (Clarke \& Ensslin 2006; Brentjens 2008). Thus, we consider a flat hadronic model, with $\omega_{\mathrm{CR}}=$ constant to $r \sim 1.5 r_{\mathrm{c}}$ and $X=$ constant for larger $r$, that produces a decrease of the brightness by a factor $8-12$ at $r \sim 1.5 r_{\mathrm{c}}$ for the range of $\left(b, B_{\mathrm{o}}\right)$ given above; this is our reference model. The energy request of the flat hadronic model is also reported in Fig. 1 by considering the conservative case of relativistic CRp only. The large energy budget of the nonthermal components is a problem for a hadronic origin of the radio halo in A2256.

This large budget and the steep spectrum of CRp unavoidably imply that there is an efficient production of $\gamma$-rays at $0.1-1 \mathrm{GeV}$ due to $\pi^{\circ}$ decay. Consequently, FERMI/GLAST observations provide an efficient and complementary way to test a hadronic origin of the halo.

In Fig. 2, we show the expected radio (left) and $\gamma$-ray (right) spectra of A2256 for different values of $B_{\mathrm{o}}$ (see caption) (models anchored to the observed $1.4 \mathrm{GHz}$ emission); we also report results for the case of a hadronic model with $s=2.4$.
We find that by assuming a hadronic origin of the radio halo and adopting the appropriate spectrum of CRp, the $\gamma$-ray upper limit obtained with EGRET observations (Reimer et al. 2003) already constrains $B_{\mathrm{o}}>2.5 \mu \mathrm{G}$. Most importantly, FERMI/GLAST should be able to detect Abell 2256 in the next few years, provided that $B_{\mathrm{o}} \leq 10-15 \mu \mathrm{G}$. This is highlighted in Fig. 3, where we show the expected photon number with $E_{\gamma} \geq 100 \mathrm{MeV}$ as a function of $B_{\mathrm{o}}$ in the case of both steep and flat hadronic models.

\section{Discussion and conclusions}

Radio halos have typical synchrotron spectral indices $\alpha \sim$ 1.2-1.3 (e.g., Ferrari et al. 2008), yet halos with steeper spectra might be more common in the Universe (e.g., Cassano et al. 2006) and present observations at $\mathrm{GHz}$ frequencies may preferentially select those halos with flatter spectra. The discovery of a few radio halos with spectral index $\alpha>1.5-1.6$ suggests that the emitting electrons are accelerated by rather inefficient mechanisms, e.g., turbulent acceleration, and constrains models, such as the hadronic one, that would require a very large energy budget to be supplied to explain the properties of these sources (e.g., Brunetti et al. 2008).

A2256 hosts the closest radio halo with a steep spectrum, of $\alpha=1.61$, that would require a spectral slope of CRp $s=3.4-3.5$ adopting the hadronic scenario; in this case, only a small fraction of the total energy-budget of supra-thermal CRp is expected to be associated with relativistic CRp. We exploit two approaches based on hadronic models: a steep model that assumes a constant fraction $X=\omega_{\mathrm{CR}} / \omega_{\mathrm{TH}}$ and a flat model that assumes $\omega_{\mathrm{CR}}=$ constant in the halo volume and $X=$ constant outside. The last one is our reference model since the observed halobrightness profile of A2256 implies a rather flat spatial distribution of CRp.

Even by considering only relativistic CRp, the hadronic model requires a large CRp-energy budget to explain the halo in A2256 for central fields $B_{0}<10 \mu \mathrm{G}$. This is a drawback of the hadronic scenario and also implies that the expected $\gamma$-ray luminosity of A2256 should be a factor of 10-20 higher than that of similar clusters hosting halos of the same radio luminosity but 


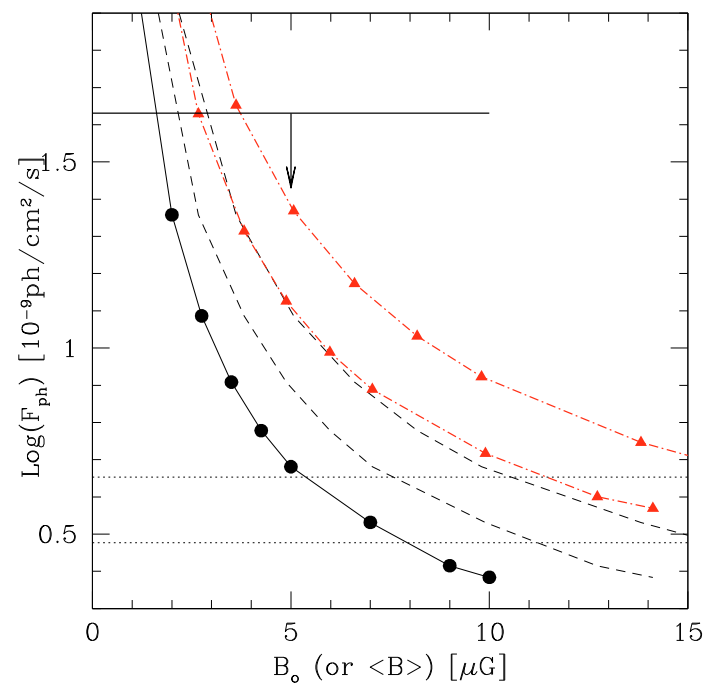

Fig. 3. Photon fluxes for $>100 \mathrm{MeV}$ are shown as a function of $B_{\mathrm{o}}$ assuming flat (red dot-dashed lines) and steep models (dashed lines) with $b=0.5$ and 1 (bottom to top). Photon flux vs. $\langle B\rangle$ for the steep model is also shown (solid line with points). EGRET upper limit (arrow) and FERMI/GLAST sensitivity-range (dotted lines) are shown.

with $\alpha \sim 1.2$. In these conditions, we show that FERMI/GLAST should be able to detect A2256.

Non-detection would either imply that the halo is not of hadronic origin, or that the magnetic field in the central cluster region is $B_{\mathrm{o}} \geq 10-15 \mu \mathrm{G}$. In the latter case, however we would concede to the ad hoc possibility that A2256 is a cluster with an unusually strong magnetic field since strong fields are presently observed only in cool-core clusters (e.g., Carilli \& Taylor 2002; Govoni \& Feretti 2004); future observations of Faraday rotation will provide complementary information about the cluster magnetic field.

On the other hand, detection of steep-spectrum $\gamma$-ray emission from A2256 would imply a hadronic origin of the halo, providing us also with an unprecedented measure of the magnetic field strength in the cluster. This will also suggest that very unusual acceleration mechanisms operate in the ICM, channeling a large fraction of the cluster energy into a population of CRp with very steep spectrum.

If the halo is generated by turbulent re-acceleration, the maximum $\gamma$-ray luminosity expected from A2256 can be estimated by requiring that the emission from secondaries matches the radio flux at the highest frequencies and is much lower than that at lower frequencies (assumed to be dominated by re-accelerated electrons) (Reimer et al. 2004; Donnert et al. 2009). This implies a $\gamma$-ray luminosity similar to that of the model with $s=2.4$ in Fig. 2 implying that detection would be possible only for weak fields, $B_{\mathrm{o}}<1 \mu \mathrm{G}$. In this case the $\gamma$-ray spectrum is much flatter than that in the case of a hadronic origin of the halo.

Acknowledgements. This work is partially supported by grants PRIN-INAF2007 and 2008, and ASI-INAF I/088/06/0.

\section{References}

Aharonian, F. A., Akhperjanian, A. G., Anton, G., et al. 2009a, A\&A, 495, 27 Aharonian, F. A., Akhperjanian, A. G., Anton, G., et al. 2009b, A\&A, 502, 437 Berezinsky, V. S., Blasi, P., \& Ptuskin, V. S. 1997, ApJ, 487, 529

Berrington, R. C., Lugger, P. M., \& Cohn, H. N. 2002, AJ, 123, 2261

Blasi, P. 2001, APh, 15, 223

Blasi, P., \& Colafrancesco, S. 1999, APh, 12, 169

Blasi, P., Gabici, S., \& Brunetti, G. 2007, Int. J. Mod. Phys. A, 22, 681

Brentjens, M. A. 2008, A\&A, 489, 69

Bridle, A. H., Fomalont, E. B., Miley, G. K., \& Valentijn, E. A. 1979, A\&A, 80, 201

Briel, U. G., Henry, J. P., Schwarz, R. A., et al. 1991, A\&A, 246, L10

Brunetti, G. 2009, RMxAC, 36, 201

Brunetti, G., \& Blasi, P. 2005, MNRAS, 363, 1173

Brunetti, G., \& Lazarian, A. 2007, MNRAS, 378, 245

Brunetti, G., Setti, G., Feretti, L., \& Giovannini, G. 2001, MNRAS, 320, 365

Brunetti, G., Blasi, P., Cassano, R., \& Gabici, S. 2004, MNRAS, 350, 1174

Brunetti, G., Giacintucci, S., Cassano, R., et al. 2008, Nature, 455, 944

Carilli, C. L., \& Taylor, G. B. 2002, ARA\&A, 40, 319

Cassano, R., \& Brunetti, G. 2005, MNRAS, 357, 1313

Cassano, R., Brunetti, G., \& Setti, G. 2006, MNRAS, 369, 1577

Clarke, T. E., \& Ensslin, T. A. 2006, ApJ, 131, 2900

Dennison, B. 1980, ApJ, 239, L93

Dermer, C. D. 1986a, A\&A, 157, 223

Dermer, C. D. 1986b, ApJ, 307, 47

Dolag, K., \& Ensslin, T. A. 2000, A\&A, 362, 151

Dolag, K., Grasso, D., Springel, V., \& Tkachev, I. 2005, JCAP, 1, 9

Donnert, J., Dolag, K., Brunetti, G., Cassano, R., \& Bonafede, A. 2009, MNRAS, in press [arXiv: 0905.2418]

Ebeling, H., Voges, W., Böhringer, H., et al. 1996, MNRAS, 281, 799

Ensslin, T. A., Biermann, P. L., Kronberg, P. P., \& Wu, X.-P. 1997, ApJ, 477, 560

Ensslin, T. A., Biermann, P. L., Klein, U., \& Kohle, S. 1998, A\&A, 332, 395

Ferrari, F., Govoni, F., Schindler, S., et al. 2008, SSRv, 134, 93

Fujita, Y., Takizawa, M., \& Sarazin, C. L. 2003, ApJ, 584, 190

Gabici, S., \& Blasi, P. 2003, ApJ, 583, 695

Govoni, F., \& Feretti, L. 2004, Int. J. Mod. Phys. D, 13, 1549

Henry, J. P., Briel, U. G., \& Nulsen, P. E. J. 1993, A\&A, 271, 413

Miller, N. A., Owen, F. N., \& Hill, J. M. 2003, AJ, 125, 2393

Miniati, F. 2003, MNRAS, 342, 1009

Moskalenko, I. V., \& Strong, A. W. 1998, ApJ, 493, 694

Myers, S. T., Baker, J. E., Readhead, A. C. S., \& Leitch, E. M. 1997, ApJ, 485, 1 Petrosian, V. 2001, ApJ, 557, 560

Pfrommer, C., \& Enßlin, T. A. 2004, A\&A, 413, 17

Pfrommer, C., Springel, V., Enßlin, T. A., \& Jubelgas, M. 2006, MNRAS, 367, 113

Reimer, O., Pohl, M., Sreekumar, P., \& Mattox, J. R. 2003, ApJ, 588, 155

Reimer, A., Reimer, O., Schlickeiser, R., \& Iyudin, A. 2004, A\&A, 424, 773

Rybicky, G. B., \& Lightmann, A. P. 1979, Radiative Processes in Astrophysics

(New York: Wiley-Interscience)

Röttgering, H., Snellen, I., Miley, G., et al. 1994, ApJ, 436, 654

Ryu, D., Kang, H., Hallman, E., \& Jones, T. W. 2003, ApJ, 593, 599

Ryu, D., Kang, H., Cho, J., \& Das, S. 2008, Science, 320, 909

Sun, M., Murray, S. S., Markevitch, M., \& Vikhlinin, A. 2002, ApJ, 565, 867

Völk, H. J., Aharonian, F. A., \& reitschwerdt, D. 1996, SSRv, 75, 279 